\title{
MicroRNA-153 functions as a tumor suppressor by targeting SET7 and ZEB2 in ovarian cancer cells
}

\author{
JUN ZHOU ${ }^{1,2,3}$, MING XIE ${ }^{3}$, YING SHI ${ }^{1,2}$, BAIHUA LUO ${ }^{1,2}$, GUANGHUI GONG $^{1,2}$, JUANNI LI $^{1,2}$, \\ JUNPU WANG $^{1,2}$, WENJIAN ZHAO ${ }^{3}$, YUAN ZI $^{3}$, XIAOYING WU ${ }^{1,2^{*}}$ and JIFANG WEN ${ }^{1,2^{*}}$ \\ ${ }^{1}$ Department of Pathology, School of Basic Medical Science, Central South University, Changsha, Hunan 410013; \\ ${ }^{2}$ Department of Pathology, Xiangya Hospital, Central South University, Changsha, Hunan 410008; \\ ${ }^{3}$ Institute of Pathology Research, Department of Pathology, Xiangnan University, Chenzhou, Hunan 423000, P.R. China
}

Received January 6, 2015; Accepted March 31, 2015

DOI: $10.3892 /$ or.2015.3952

\begin{abstract}
The overall goal of the present study was to find and validate unidentified miRNAs that regulate epithelialmesenchymal transition (EMT) and proliferation in ovarian cancer. Furthermore, we demonstrate that the high expression of miR-153 in human epithelial ovarian cancer (EOC) is associated with better survival. The mean expression level of miR-153 in ovarian cancer was significantly lower than in the adjacent carcinoma tissue. In the present study, we report that miR-153 are negative regulators of SET7 and ZEB2, miR-153 regulates SET7/ZEB2 expression and promotes SET7/ZEB2 mRNA degradation. Further, confirmed by reporter assays, SET7/ZEB2 are downstream targets of miR-153 directly bound to the 3 ' untranslated region (3'-UTR). Clone formation and wound-healing assay as well as Transwell assay proved that silencing of SET7 or ZEB2 partially abolished the enhancement of cell proliferation and invasion induced by downregulated miR-153. SET7 and ZEB2 are negatively correlated with miR-153 expression in human ovarian cancer and indicated a worse survival. Considering the role of SET7 and ZEB2 in EOC, it is important to clarify how the expression of SET7 and ZEB2 are regulated. Based on our results miR-153 inhibits proliferation and suppresses EMT and the invasive potential of ovarian cancer cells through downregulation of SET7 and ZEB2, supporting the pursuit of miR-153 as a potential target for ovarian cancer intervention.
\end{abstract}

\section{Introduction}

MicroRNAs are a cluster of conserved, small, non-coding single-stranded RNAs (18-25 nucleotides), the function of

Correspondence to: Professor Jifang Wen or Professor Xiaoying Wu, Department of Pathology, School of Basic Medical Science, Central South University, Changsha, Hunan 410013, P.R. China

E-mail: jifangwen001@163.com

E-mail: xiaoyingwuchangsha@163.com

"Contributed equally

Key words: microRNA-153, tumor suppressor, SET7, ZEB2 which are to modulate gene expression by binding to the target gene with complementary recognition sequences of $3^{\prime}$ untranslated regions (3'-UTRs), leading to target mRNA degradation or negatively regulating translational efficiency (1-3). Growing amounts of evidence indicate that miRNA expression is altered in almost all types of cancer (4-7). The deregulation of miRNAs plays an important role in various cellular processes, such as proliferation, the cell cycle, apoptosis and metastasis contributing to tumorigenesis $(8,9)$.

Despite growing knowledge in understanding ovarian cancer etiology, of which more than $85 \%$ are of epithelial origin. Epithelial ovarian cancer (EOC) remains the most lethal form of gynecological malignancy in the USA (10-12). EOC are classified into distinct histologic types including serous ( $60 \%$ of all cancers), mucinous, endometrioid and clear cell (less common histologies) $(13,14)$. Shih and Kurman divided EOC into 2 types (15), type I includes low-grade serous, low-grade endometrioid, mucinous and clear cell carcinomas, and type II EOC includes the high-grade types (15).

The molecular mechanisms that contribute to the tumor progression such as proliferation and metastasis still need further exploring. We focused on ZEB2/SIP1, which are potent repressors of E-cadherin expression, through binding to $\operatorname{CACCT}(\mathrm{G})$ motif in the E-cadherin promoter (16) and considered to be a key factors of epithelial-mesenchymal transition (EMT) (17). EMT are believed to be the initial step of tumor metastasis, during EMT epithelial cells lose their differentiated epithelial characteristics, and acquire mesenchymal features, such as motility, and invasiveness (18). ZEB2 plays important role in the development of a variety of cancers, such as gastric, squamous, breast, non-small cell lung and ovarian carcinomas (19-21). Besides E-cadherin, other genes coding for crucial proteins such as plakophilin 2 involved in tight junctions were found to be repressed by ZEB2 (17). Recently, vimentin was reported to be upregulated by ZEB2, another mesenchymal marker, which is associated with breast tumor cell migration and invasion in a study by Bindels et al (22).

SET domain containing 7 (SET7) is a lysine methyltransferase with the function of methylation histone and 
non-histone proteins. By which it has diverse functions in transcription regulation, diabetes, DNA repair, tumor metastasis, proliferation and genome integrity (23-26). The activity of SET7 is tightly controlled through post-translational modifications, including ubiquitination, phosphorylation and sumoylation $(27,28)$. Yet, the role of microRNAs in the regulation of SET7 has not been discovered.

Considering EMT is a process regulated by numerous factors, it is reasonable to hypothesize that different miRNAs are involved in the regulation. However, although the miR-200 familys in EMT have been well discussed, the roles of other miRNAs are still poorly understood $(29,30)$. Thus, there is an urgent need to better understand the role of miRNA in EOC to develop novel therapeutics for this disease.

\section{Materials and methods}

Cell culture. Human ovarian serous cystic adenocarcinoma cell line OVCAR3 and human serous papillary cystic adenocarcinoma cell line SKOV3 were purchased from the American Tissue Type Collection (Manassas, VA, USA). Both cell lines are suitable transfection hosts. Normal epithelial ovarian cells (NOE095 and HOSEpiC) were obtained from the Chinese Academy of Sciences. All cells were cultured in RPMI-1640 medium supplemented with $10 \%$ fetal calf serum (Gibco), $100 \mathrm{IU} / \mathrm{ml}$ penicillin and $100 \mathrm{IU} / \mathrm{ml}$ streptomycin. These cell lines were grown at $37^{\circ} \mathrm{C}$ in a humidified atmosphere with $5 \%$ $\mathrm{CO}_{2}$. For the transfections, cells were grown to $70 \%$ and transfected with plasmids using Lipofectamine 2000 (Invitrogen, USA) according to the manufacturer's recommendation.

Clinical specimens. A total of 60 cases of ovarian cancer specimens and their adjacent normal tissues were obtained from Xiangya hospital. The age ranged from 28 to 55, the average was $(34 \pm 8)$. The patients who had received radiation or chemotherapy prior to the surgery were excluded. The tissue samples were stored in liquid nitrogen. The survival times were calculated from the operation day to death, via the evaluation of recurrence and metastasis. Samples were selected from patients with complete clinicopathologic information. The study was approved by the Xiangya hospital ethics committee.

Antibodies and siRNAs. Primary antibodies used were rabbit anti-ZEB2 (Sigma-Aldrich), mouse anti-SET7 (Abcam) and rabbit anti $\beta$-actin (Sigma-Aldrich). $\beta$-actin was used for normalization. The small interfering RNAs (siRNA) targeting human ZEB2, SET7, control siRNA were from (Sigma-Aldrich), miR-153 mimics were synthesized by Shanghai GeneChem Inc. (Shanghai, China), miR-153 inhibitors and inhibitor negative control (anti-miR-NC) were obtained from Dharmacon.

Transfection of miRNA mimics and inhibitors. Cells were seeded in a 6-well plates and transfected with synthetic miRNA-153 mimics or miR-153 inhibitors or miR-control or inhibitor-control at $3 \times 10^{5}$ cells/well with a final concentration of $100 \mathrm{nM}$ using Lipofectamine 2000, according to the instructions provided by the manufacturer. Total RNA were collected 2 days post-transfection, and protein 3 days, transfection efficiency was monitored by qRT-PCR. The sequences of miR-153 were as follows: sense, 5'-UUGCAUAGUCACAAAAGUGA
UC-3' and antisense, 5'-GAUCACUUUUGUGACUAUGC AA-3'. For miRNA analysis, real-time PCR was performed using PrimeScript ${ }^{\circledR}$ miRNA RT-PCR kit (Takara) according to the manufacturer's instructions. All miRNA data are expressed relative to a U6 small nuclear RNA from the same sample.

RNA extraction and real-time PCR. Total RNA was extracted using TRIzol (Invitrogen), and total RNA was extracted using a High Pure miRNA Isolation kit (Roche) according to the manufacturer's instructions, for analysis of SET7 and ZEB2 messenger RNA (mRNA) expression, RNA were reverse transcribed to cDNA using $1 \mu \mathrm{g}$ of total RNA with M-MLV reverse transcriptase, according to the manufacturer's instructions. The specific primers for SET7, ZEB2 and $\beta$-actin were as follows: SET7 forward, 5'-CCTCACTTTGAACTGATGCC-3' and reverse, 5'-CAGCAACATAAACCCTTTCTG-3'; ZEB2 forward, 5'-AGGAGCTGTCTCGCCTTG-3' and reverse, 5'-G GCAAAAGCATCTGGAGTTC-3'; and $\beta$-actin forward, 5'-T GGCACCCAGCACAATGAA-3' and reverse, 5'-CTAAGTC ATAGTCCGCCTAGAAGCA-3'; $\beta$-actin served as the internal control.

Plasmid construction and luciferase assays. The wild-type and mutant SET7 or ZEB2 3'-UTR were amplified by PCR and cloned in pMIR-Report (Ambion) to generate a series of reporter constructs with firefly luciferase. The luciferase assays were performed in OVCAR3 cells as described previously, OVCAR 3 cells treated with control, miR-153 mimics, or miR-153 inhibitors were transfected with wild-type or mutants of SET7 or ZEB2 3'-UTR luciferase reporters along with Renilla plasmid, using Lipofectamine LTX-Plus (Invitrogen). Forty-eight hours after transfection, the firefly and Renilla luciferases were assayed according to the manufacturer's protocol (Promega), and the firefly luciferase activity was normalized to that of Renilla luciferase. Each experiment was repeated in triplicate. The amount of DNA was kept constant by addition of empty vector in each transfection.

Western blotting. Cells were harvested $48 \mathrm{~h}$ after infection in each group and protein concentration was determined using the bicinchoninic acid (BCA). Subsequently, $30 \mu \mathrm{g}$ protein were run by $10 \%$ SDS-PAGE gel and transferred to NC membranes. $\mathrm{NC}$ membranes were blocked by $5 \%$ skim milk for $1 \mathrm{~h}$, then incubated overnight at $4^{\circ} \mathrm{C}$ with primary antibodies, including ZEB2 (1:1,000), SET7 $(1: 1,000)$ and $\beta$-actin $(1: 500)$. Goat anti-rabbit secondary antibodies $(1: 5,000)$ or goat anti-mouse secondary antibodies $(1: 3,000)$ and Western Blotting Luminol Reagent (Santa Cruz Biotechnology) was used to visualize the protein bands.

Colony formation assay. A total of 5,000 cells were inoculated in 6-well plates in $1.5 \mathrm{ml}$ of RPMI-1640 medium supplemented with $10 \%$ fetal bovine serum (FBS), after the infection for 3 days. The medium was changed at the interval of 3 days; after 10 days of culturing, the colonies (which were a cluster of cells seen under the microscope) formed, the plates were washed with phosphate buffered-saline (PBS) and fixed in $4 \%$ paraformaldehyde at $37^{\circ} \mathrm{C}$ for $30 \mathrm{~min}$, after which the colonies were stained with $0.005 \%$ crystal violet (Sigma) in PBS for $15 \mathrm{~min}$, washed and then air-dried. The colonies were counted 
using microscopy (Olympus, Tokyo, Japan). This experiment was performed in triplicate.

Wound-healing assay. SKOV3 cells were seeded in 12-well plates and cultured to form a confluent monolayer. Wounds (2-mm) were made with a sterile plastic scraper, the floating cells were washed away three times with PBS. Images were captured at indicated time points after wounds were made. After incubation in a serum-free medium for $24 \mathrm{~h}$, to exclude the impact of serum medium on proliferation, cultures were observed and images were captured under a microscope. At least five randomly chosen areas were measured, and repeated three times.

Transwell invasion assay. The Transwell invasion assays were performed using the Transwell chamber $(8-\mu \mathrm{m}$ pore size, for 24-well plates; Millipore) with a Matrigel-coated filter. OVCAR3 cells were transfected with control, miR-153 mimics or miR-153 inhibitors with/without ZEB2 cDNA lacking 3'-UTR. A total of $100 \mu 1$ of the cell suspension (50,000 cells) with serum-free medium was added to the upper chamber of the Transwell, and $500 \mu \mathrm{l}$ of RPMI-1640 containing 10\% FBS was added to the lower compartment. After incubation for $24 \mathrm{~h}$, cells on the upside were removed using cotton swabs, and the invasive cells on the lower side were fixed, stained with $0.1 \%$ crystal violet solution, and counted using light microscopy. The experiments were repeated three times.

Bioinformatics analysis and statistical analysis. The bioinformatics method was used to predict the potential target genes of miR-153. The microRNA.org (http://www.microrna.org/ microrna/), miRDB (http://mirdb.org/cgi-bin/) and TargetScan (http://www.targetscan.org/) databases were used, SET7 and ZEB2 was considered as the candidate gene.

Statistical significance was assessed by comparing mean values $( \pm \mathrm{SD})$ using a Student's t-test for independent groups. $\mathrm{P}<0.05$ were considered to indicate a statistically significant result. One-way analysis of variance (ANOVA) with SNK-q test for multiple comparisons was used to analyze the data from the Transwell migration assay and western blotting using SPSS 15.0 software. ${ }^{*} \mathrm{P}<0.05,{ }^{* *} \mathrm{P}<0.01$.

\section{Results}

miR-153 is a potential tumor suppressor in ovarian cancer. In order to explore the role of miR-153 during the progress of ovarian cancer, we first enrolled 60 ovarian cancer patients. They were divided into two groups based on their miR-153 expression levels: those with less than median of miR-153 expression levels and those with more than or equal to median of miR-153 expression levels. The higher expression of miR-153 indicates a better overall 5-year survival (the hazard ratio, 2.4183, $\mathrm{P}<0.05$ ) Kaplan-Meier curves were drawn, the details are shown in Fig. 1A.

To investigate the role of miR-153 in human ovarian cancer, the expression of miR-153 was compared using qRT-PCR in ovarian cancer samples and corresponding noncancerous tissues. The results showed that expression level of miR-153 in ovarian cancer was significantly lower (median, -1.5099; max, -0.97469; min; -2.0385) than in non-cancerous tissues (median, 1.1019]; max, 3.3357; min, -1.2377) $(\mathrm{P}<0.01$, Fig. 1B). Growth assays in cells transfected with miR-control or miR-153, OVCAR3 cells with miR-153 showed an obvious growth inhibition (Fig. 1C). To further explore the miR-153 impact on migration and invasion, a wound-healing assay was conducted. As shown in Fig. 1D, $24 \mathrm{~h}$ after the wound were created and measured, cells with miR-153 were significantly impaired in wound recovery, compared with the control cells. To further assess the effects on cell invasion, Transwell assay was performed. Migrated cells infected with miR-153 counts for one third of those infected with miR-control (Fig. 1E), the above data show miR-153 functions as a potential tumor suppressor in ovarian cancer.

miR-153 regulates the expression and $m R N A$ degradation of the target gene SET7. As reported, miRNAs silence genes either by translational inhibition or mRNA destabilization $(31,32)$. Connecting with the bioinformatic prediction, with analyses based on three public algorithms (TargetScan, PicTar and miRanda), indicated that the SET7 3'-UTR harbors two potential miR-153 target sites (positions 46-52 and 4732-4738), both positions are highly conserved regions in different species (Fig. 2A and B). These sites are completely complementary to the human miR-153 heptamer motif 5'-UGCAUAG-3'.

To prove the proposition that SET7 is a downstream target of miR-153, reporter assays were carried in OVCAR3 cells. Luciferase gene is driven by wild-type or mutated SET7 3'-UTR sequences, including the full-length or three deletions of the wild-type SET7 3'-UTR or 2 mutants (with three point mutations) of SET7 3'-UTR (Fig. 2C, left panel). The results demonstrated $>60 \%$ reduction in luciferase activity with the full-length wild-type (wt-1) SET7 3'-UTR in OVCAR3 cells transfected with miR-153. Consistent with our prediction, the site A including the position 46-52 or site $\mathrm{C}$ including the position 4732-4738 plays a more important role in the binding of miR-153 to SET7 3'-UTR, as there was more evident decrease in luciferase activity with wt-2, wt-4 compared with that of wt-3 (Fig. 2C, right panel), point mutation of mut-1 with site A and mut- 2 with site $\mathrm{C}$ disrupted miR-153 repressive activity, further indicated the binding of miR-153 to sites A and C of SET7 3'-UTR was specific. Taken together, these results suggest that miR-153 binds to SET7 3'-UTR. We then investigated whether binding to the SET7 3'-UTR has an effect on the expression of SET7 mRNA and protein. For this purpose, OVCAR3 and SKOV3 cells were transfected with control or miR-153, the results showed that miR-153 overexpression was decreased by more than half in SET7 mRNA in the two cell lines, respectively (Fig. 2D, left panel), and western blot analysis showed an obvious reduction of SET7 protein expression (Fig. 2D, right panel). Consistently, OVCAR3 and SKOV3 cells transfected with miR-153 inhibitors resulting in an increase in SET7 mRNA by real-time quantitative PCR (qPCR) (Fig. 2E, left panel) and a significant rise in SET7 protein expression by western blotting (Fig. 2E, right panel). To further evaluate the impact of miR-153 on SET7 mRNA stability, OVCAR3 and SKOV3 cells treated with transcription inhibitor actinomycin D were then transfected with miR-153 mimics or miR-153 inhibitors. Real-time qPCR showed that miR-153 overexpression led to a reduced SET7 mRNA 

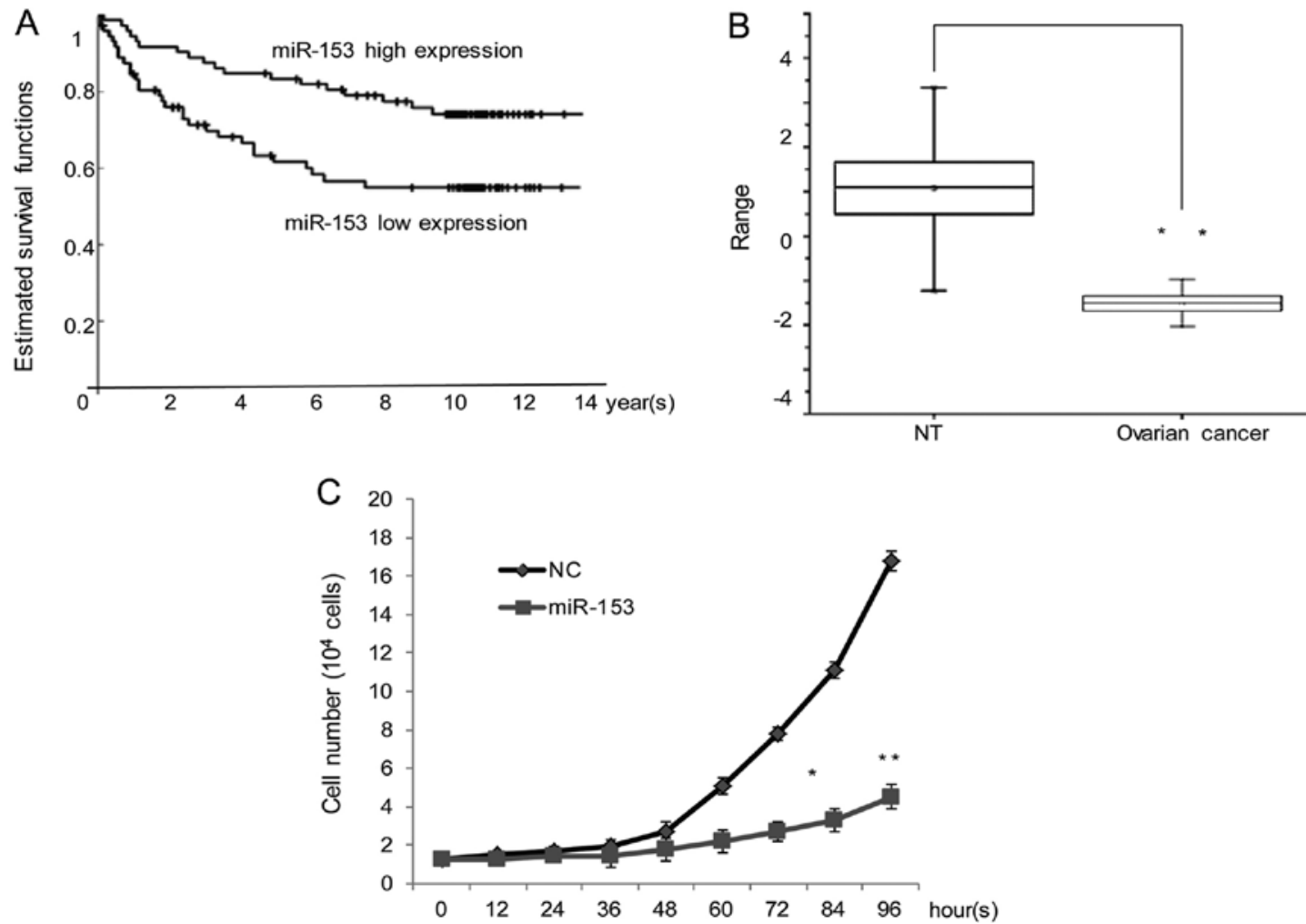

D
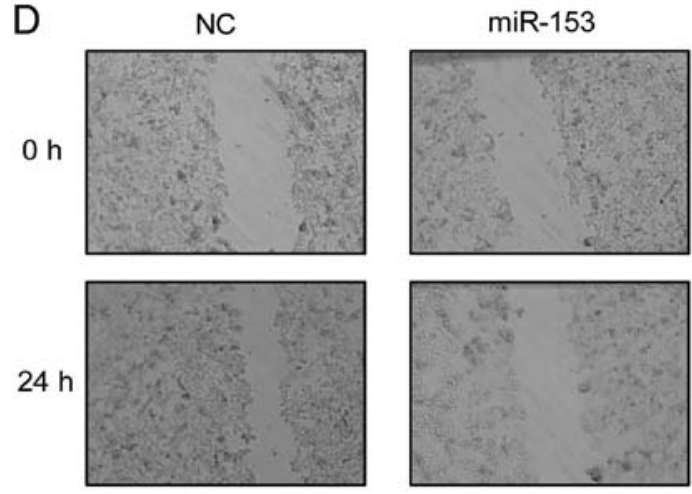

E

NC

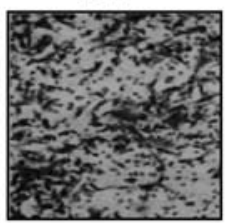

$\operatorname{miR}-153$

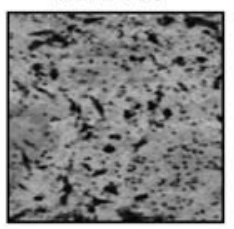

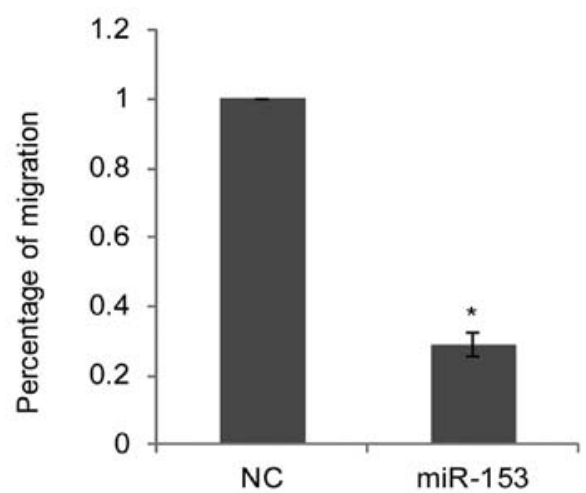

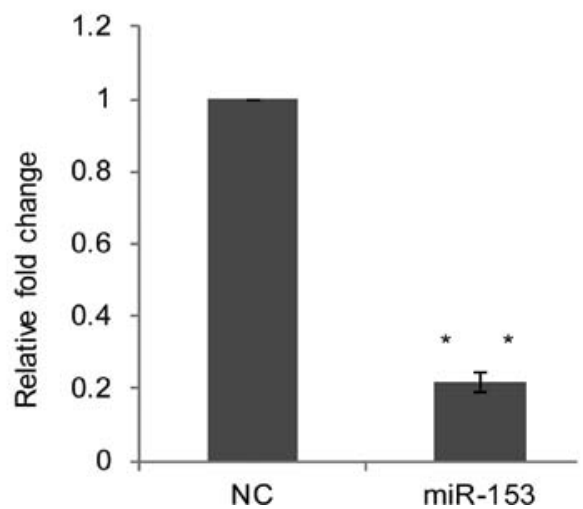

Figure 1. miR-153 is a potential tumor suppressor in ovarian cancer. (A) The expression of miR-153 was assessed to evaluate the impact of overall 5-year survival, Kaplan-Meier curves were drawn, Cox proportional hazards regression was used to test the prognostic significance of factors in univariate and multivariate models. $\mathrm{P}<0.05$ was considered significant. (B) The expression of miR-153 was determined by real-time PCR in 60 pairs of EOC and adjacent non-cancerous tissue (NT). Error bars represent standard error of the mean Student's t-test, ${ }^{* *} \mathrm{P}<0.01$. (C) miR-153 inhibits cellular proliferation. OVCAR3 cells transfected with NC or miR-153 were subjected to growth curve analysis. Equal numbers of cells were mixed, and seeded into replicate plates. The numbers of cells were counted every $12 \mathrm{~h}$. Error bars represent the mean $\pm \mathrm{SD}$ for triplicate measurements, ${ }^{*} \mathrm{P}<0.05 ;{ }^{* *} \mathrm{P}<0.01$. (D) miR-153 inhibits cellular migration. OVCAR3 cells transfected with control for NC or miR-153 were subjected to wound-healing analysis. Wound recovery was photographed $24 \mathrm{~h}$ post-infection. Migrated cells in migration and invasion assays were measured; ${ }^{*} \mathrm{P}<0.05{ }^{*}{ }^{* *} \mathrm{P}<0.01$. (E) OVCAR3 cells were transfected with NC or miR-153, after $48 \mathrm{~h}$ transfection, cells were starved for $18 \mathrm{~h}$ before cell invasion assays were performed. The invaded cells were stained and counted, representative images are shown. Statistical analysis are presented as fold-change over the vector. Error bars are the mean \pm SD for triplicate measurements. ${ }^{*} \mathrm{P}<0.05 ;{ }^{* *} \mathrm{P}<0.01$. EOC, epithelial ovarian cancer. 
A

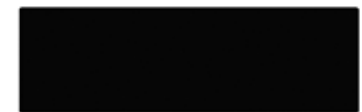

Predicted consequential pairing of target region(top) and miRNA(bottom)

Position 46-52 of SETD7 3'UTR hsa-miR-153

\section{5'...AAUAGAAACUUGGAU--CUAUGCAC..}

CUAGUGAAACACUGAUACGUU

B

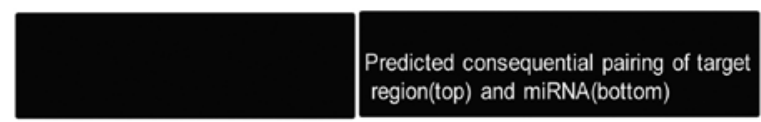

Position 4732-4738 of SETD7 3'UTR 5'...CUCAGCUCUGGAAGACUAUGCAC hsa-miR-153

II III I I

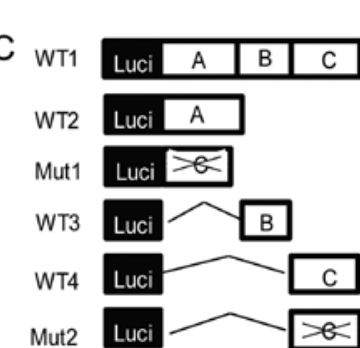

3' CUAGUGAAACACUGAUACGUU

Mut2
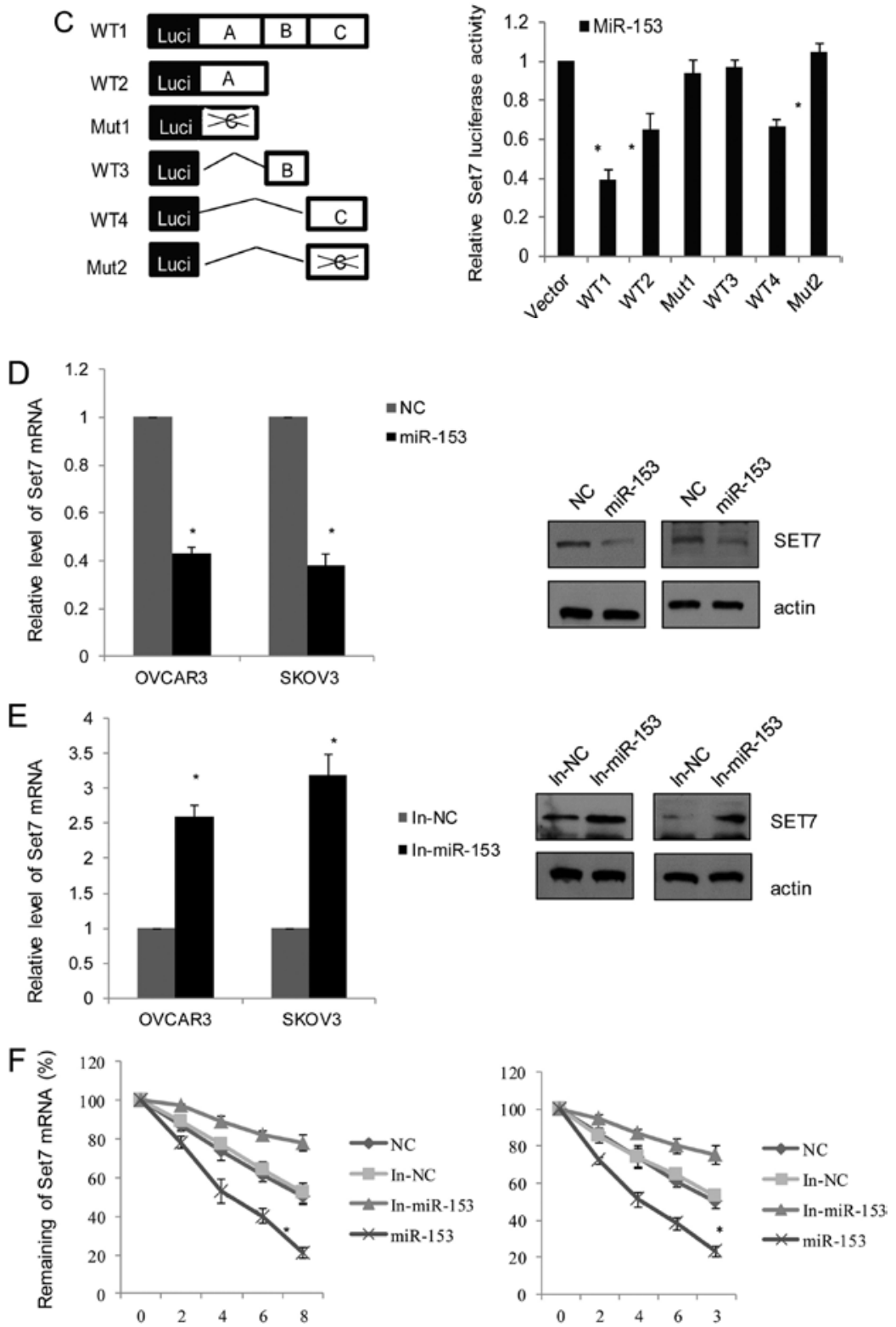

Figure 2. miR-153 regulates the expression and mRNA degradation of the target gene SET7. (A) Potential targets for miR-153 were predicted through PicTar, TargetScan and miRanda. TargetScan represent the position 46-52 of SET7 3'-UTR was a highly conserved sequence considered to be one of the candidate targets for miR-153. (B) Position 4732-4738 of SET7 3'-UTR was predicted as another target with high score. The miR-153 seed sequence (5-UGCAUAG-3) and the corresponding complementary sequences of SET7 3'-UTR are shaded. (C) The diagram shows luciferase reporter constructs for full-length wildtype (wt-1), deletions of wild-type (wt-2, -3 and -4), and two point mutants (mut-1 and 2) of SET7 3'-UTR. Luciferase reporter assays were performed to identify the binding of miR-153 to SET7 3'-UTR. OVCAR3 cells were co-transfected with negative control (NC) or miR-153 mimics (miR-153) together with the luciferase gene driven by different SET7 3'-UTR sequences. The normalized luciferase activity was the control. Each bar represents the mean \pm SD for triplicate experiments. (D) OVCAR3 or SKOV3 cells were transfected with control or miR-153 mimics for $48 \mathrm{~h}$. The endogenous SET7 mRNA and protein expression were measured by real-time qPCR or western blotting. The relative level of SET7 mRNA was normalized to $\beta$-actin. (E) Respectively, OVCAR3 or SKOV3 cells were transfected with control-inhibitors, or miR-153 inhibitors, the relative mRNA and protein expression were measured. (F) miR-153 destabilizes SET7 mRNA. OVCAR3 and SKOV3 cells were transfected with control, miR-153 mimics or miR-153 inhibitors for $36 \mathrm{~h}$ and then treated with actinomycin D $(5 \mu \mathrm{g} / \mathrm{ml})$ for the indicated times. The endogenous SET7 mRNA was measured by qPCR, and normalized to $\beta$-actin. Each bar represents the mean \pm SD for triplicate experiments. 3'-UTR, 3' untranslated region. 
half-time (from 8 to 4 h) while an increased SET7 mRNA half-time was due to miR-153 depletion (Fig. 2F), further supporting the fact that miR-153 represses SET7 expression through destabilization of SET7 mRNA.

ZEB2 is a downstream target of miR-153 directly binding to its 3'-UTR. To identify ZEB2 as a target of miR-153, using bioinformatics method as the way to discuss SET7, also two highly conserved regions were found located in the ZEB2 3'-UTR (positions 689-695 and 191-197), (Fig. 3A and B). The motif 5'-UGCAUA-3' of human miR-153 are fully complementary with ZEB2 3'-UTR.

Further, reporter assays were carried out in OVCAR3 cells with the luciferase gene driven by either four wildtype or two mutated ZEB2 3'-UTR sequences (Fig. 3C, left panel), the results indicated the sites A (including position 191-197) and B (including position 689-695) play a more important role in the binding of miR-153, as there were more evident decreases in luciferase activity with wt-2, and wt-3 compared with that of wt -4 . Further, the relative mutation (mut-1 with site A and mut-2 with site B) blocked miR-153 repressive activity on reporter gene expression (Fig. 3C, right panel). Real-time quantitative PCR and western blot analysis showed OVCAR3 and SKOV3 cells with miR-153 overexpression (Fig. 3D) or miR-153 inhibition (Fig. 3E), the mRNA or protein level of ZEB2 reduced or increased. The influence of ZEB2 mRNA stability by miR-153 were further measured, as well as SET7, miR-153 overexpression also led to a reduced ZEB2 mRNA half-life (from 12 to $6 \mathrm{~h}$ ) while miR-153 inhibition was associated with an increased ZEB2 mRNA half-life (Fig. 3F). As a conclusion, the miR-153 represses ZEB2 expression may also be through destabilization of ZEB2 mRNA, similarly to SET7.

miR-153 suppresses proliferation and invasive potential of ovarian cancer cells by downregulating SET7 and ZEB2. Furthermore, to examine whether miR-153 regulated EOC cell line proliferation by suppressing SET7 in vitro, proliferation assay was detected by colony formation assay. OVCAR3 cell lines were transfected with NC (mimics negative control), miR-153 mimics, miR-153 mimics plus SET7 overexpression, miR-153 mimics plus ZEB2 overexpression, or inhibitor NC, miR-153 inhibitor, miR-153 inhibitor plus siSET7, miR-153 inhibitor plus siZEB2, respectively. The transfection efficiency was validated using qRT-PCR (Fig. 4A). The colony formation assay showed that miR-153 overexpression or downregulation affected cell proliferation, the upregulation of miR-153 inhibition the colony formation was reduced by SET7 overexpression and the downregulation of miR-153 expression promoted proliferation ability could be blocked by the additional SET7 knockdown (Fig. 4B). The above data suggested that miR-153 that could inhibit OVCAR3 cell line proliferation by the suppression of SET7 in vitro.

We investigated the effect of miR-153 overexpression on cell lines invasion using wound healing or Transwell invasion assay (Fig. 1D and E). Considering ZEB2 as a key regulator in EMT (20), and as a potential target of miR-153, in the present study, we further proved the effect of gain-of-function or loss-of-function of miR-153 and ZEB2 as downstream target genes on the invasive potential in SKOV3 cells by woundhealing assays.

The results showed that miR-153 inhibitor-treated cells were more efficient in wound healing, whereas cells treated with miR-153 inhibitor plus ZEB2, loss of function was resistant to wound healing to certain degree, compared with miR-153 inhibitor only, the representative images (left panel) and the overall tendency was compared between NC (mimics negative control), miR-153 mimics, miR-153 mimics plus ZEB2 overexpression, or inhibitor NC, miR-153 inhibitor, miR-153 inhibitor plus siZEB2, respectively (Fig. 4C). In addition, Transwell invasion assays showed that miR-153 knockdown promoted the invasion by 4 -fold. Moreover, miR-153-suppressed EMT of EOC cancer cells was probably mediated by ZEB2, as downregulation of ZEB2 was able to rescue the effect of knockdown miR-153 on tumor migration and invasion, however, the overexpression of miR-153 was blocked by ZEB2 upregulation (Fig. 4D). Together, these results support the argument that miR-153 suppresses EMT and invasion of ovarian cancer cells and is at least partially, mediated by targeting ZEB2.

SET7 and ZEB2 are negatively correlated with miR-153 expression in human ovarian cancer and indicate a worse survival. Moreover, an inverse correlation was observed between miR-153 and SET7 (R=0.7141; $\mathrm{P}<0.001$; Fig. 5A, left panel), or miR-153 and ZEB2 ( $\mathrm{R}=0.7669$; $\mathrm{P}<0.0001$; Fig. 5A, right panel) in ovarian cancer samples. To further explore the role of miR-153 and SET7 or ZEB2 expression in ovarian cancer tumorigenesis, the expression of miR-153 and SET7 or ZEB2 was examined in two ovarian cancer cell lines (OVCAR3 and SKOV3) and two normal ovarian cell lines using qRT-PCR. As shown in Fig. 5B, left panel, the expression of miR-153 was substantially reduced in OVCAR3 and SKOV3 cell lines derived from ovarian cancer, compared with the normal NOE095 and HOSEpiC cell lines. However, the expression of SET7 and ZEB2 was significantly increased in OVCAR3 and SKOV3, comparing with NOE095 and HOSEpiC. The tendency was negatively correlated with miR-153 (Fig. 5B, middle and right panel). The result of correlation analysis suggested a potential functional link of miR-153 and SET7, or miR-153 and ZEB2. Further, the patients collected were divided into different groups, based on the single SET7 or ZEB2 expression level, Kaplan-Meier curves were drawn (Fig. 5C). As the data showed, the higher expression of SET7 indicates a worse 5-year survival (the hazard ratio, 2.3353, P<0.05) (Fig. 5C, upper panel). Similarly, patients with more than, or equal to median, of ZEB2 expression also had a worse 5-year survival (the hazard ratio, 2.4134, $\mathrm{P}<0.05$ ) (Fig. 5C, lower panel). These results indicated that by downregulation of SET7 and ZEB2, miR-153 may be involved in the proliferation and metastasis of ovarian cancer.

\section{Discussion}

microRNAs have been reported to be upregulated or downregulated in a various disease states and specific cell types, the action of microRNA as either oncogenes or tumor suppressors depended on the target gene $(33,34)$. By regulating the target genes with binding to their 3'-UTR, miRNAs have been 


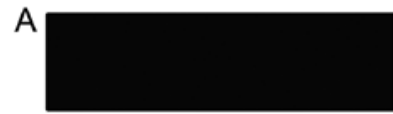

Predicted consequential pairing of target region(top) and miRNA(bottom)

Position 191-197 of ZEB2 3'UTR 5 '...UGUUAAAAACUGCAUUAUGCAAA. hsa-miR-153

3' CUAGUGAaAaCACUgAUACGUU

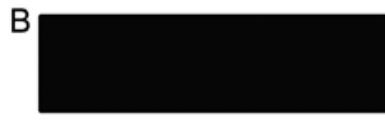

Predicted consequential pairing of target region(top) and miRNA(bottom)

Position 689-695 of ZEB2 3'UTR hsa-miR-153

5'...GUUUUAAAAGCCUUAUAUGCAAA.

3' CUAGUGAAAACACUGAUACGUU

C
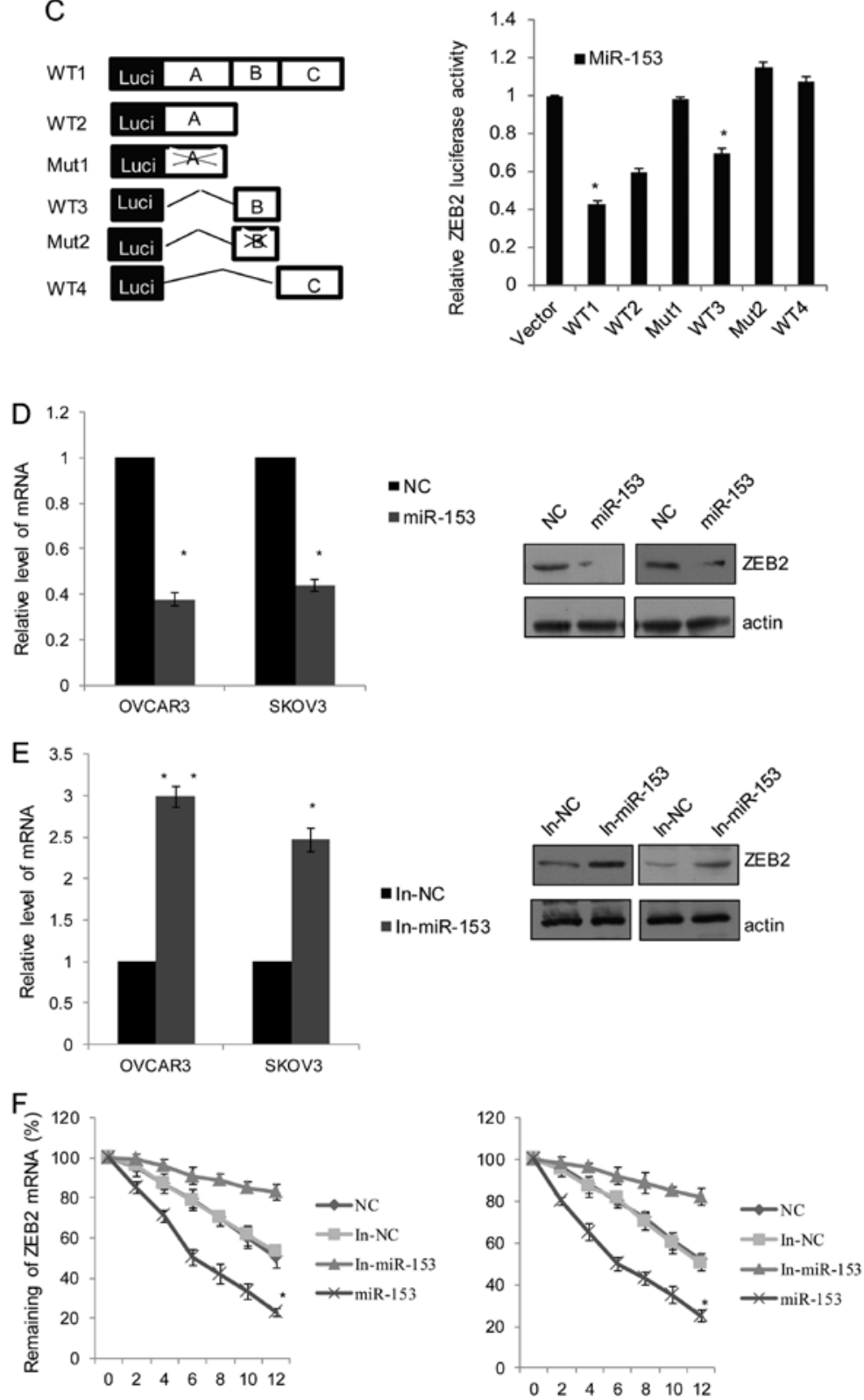

Figure 3. ZEB2 is a downstream target of miR-153 directly binding to its 3'-UTR. (A) TargetScan representing position 191-197 ZEB2 3'-UTR was a highly conserved sequence and considered to be one of the candidate targets for miR-153. (B) Position 689-695 of ZEB2 3'-UTR was also a highly conserved sequence and considered to be one of the candidate targets for miR-153. (C) Luciferase reporter assays were performed in OVCAR3 cells that co-transfected with negative control (NC) or miR-153 together with the luciferase gene driven by either wild-type or mutated ZEB2 3'-UTR sequences, including the full-length and three deletions of the wild-type ZEB2 3'-UTR (wt-1, -2, -3 and -4) as well as two mutants of ZEB2 3'-UTR (mut-1 and -2). The normalized luciferase activity in the control group was set as relative luciferase activity. Each bar represents the means \pm SD for triplicate experiments. P-values were determined by Student's t-test. (D) The endogenous ZEB2 mRNA (left panel) and protein expression (right panel) were measured by real-time qPCR and western blotting, respectively. OVCAR3 or SKOV3 cells were transfected with control, miR-153 mimics for $48 \mathrm{~h}$. Each bar represents the mean \pm SD for triplicate experiments. (E) OVCAR3 or SKOV3 cells were transfected with control-inhibitors, miR-153 inhibitors for $48 \mathrm{~h}$, the endogenous ZEB2 mRNA (left panel) and protein expression (right panel) was measured. Each bar represents the mean \pm SD for triplicate experiments. (F) miR-153 destabilizes ZEB2 mRNA. For the methods see Fig. 2E. The endogenous ZEB2 mRNA was measured by qPCR, and the relative level of ZEB2 mRNA was normalized to $\beta$-actin. Each bar represents the mean \pm SD for triplicate experiments. ${ }^{*} \mathrm{P}<0.05 ;{ }^{* *} \mathrm{P}<0.01 .3^{\prime}$-UTR, 3 ' untranslated region. 

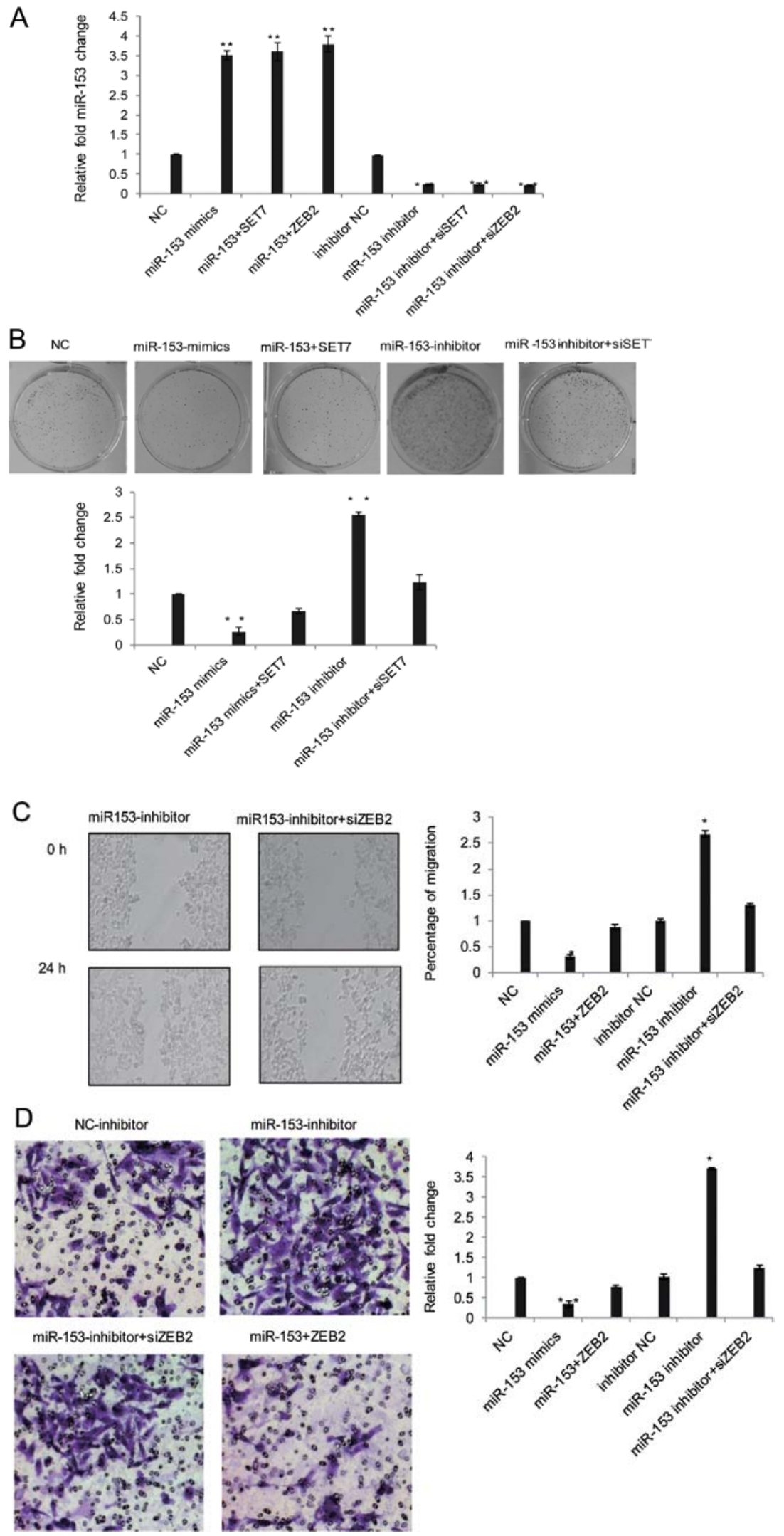

Figure 4. miR-153 suppresses proliferation and invasive potential of ovarian cancer cells by downregulating SET7 and ZEB2. (A) The results of miR-153 expression in cell lines transfected with NC (mimics negative control), miR-153 mimics, miR-153 mimics plus SET7 overexpression, miR-153 mimics plus ZEB2 overexpression, or inhibitor NC, miR-153 inhibitor, miR-153 inhibitor plus siSET7, miR-153 inhibitor plus siZEB2 were validated using qRT-PCR. (B) Clone formation assay were carried out with the relative transfection, then stained with crystal violet. Representative images are shown. Statistical analysis are presented as fold-change over the vector. Error bars are the mean $\pm \mathrm{SD}$ for triplicate measurements. ${ }^{*} \mathrm{P}<0.05 ;{ }^{* *} \mathrm{P}<0.01$. (C) Wound healing assay was performed as described in the Materials and methods. Cells treated with miR-153 mimics, miR-153 mimics plus ZEB2, miR-153 inhibitor, miR-153 inhibitor plus siZEB2, NC and inhibitor NC. All experiments were performed in triplicate and presented as mean \pm SD. (D) Transwell assay was performed as described in the Materials and methods. Cells treated with miR-153 mimics, miR-153 mimics plus ZEB2, miR-153 inhibitor, miR-153 inhibitor plus siZEB2, NC and inhibitor NC. All experiments were performed in triplicate and are presented as mean $\pm \mathrm{SD}$. ${ }^{*} \mathrm{P}<0.05 ;{ }^{* *} \mathrm{P}<0.01$ indicate significant difference compared with control group. 

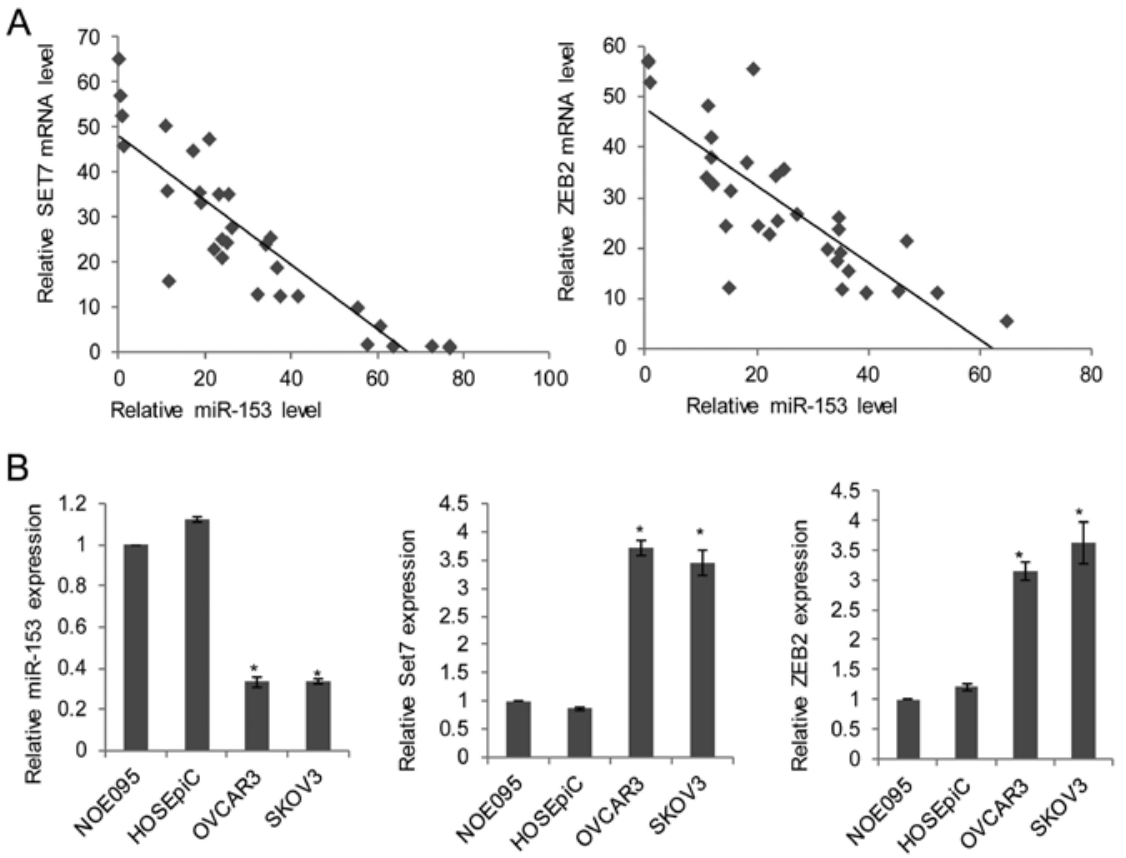

C
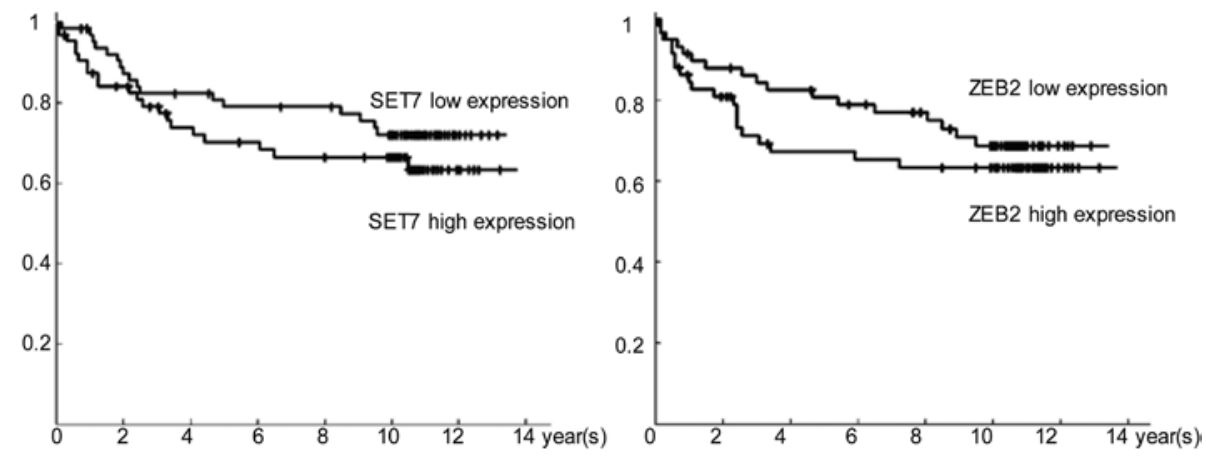

Figure 5. SET7 and ZEB2 are negatively correlated with miR-153 expression in human ovarian cancer and indicate a worse survival. (A) Negative correlation was found between RNA expression of miR-153 and SET7, or miR-153 and ZEB2 in ovarian tumor samples. miR-153 and SET7 (R=0.7141; P $<0.001$; left panel), miR-153 and ZEB2 (R=0.7669; $\mathrm{P}<0.0001$; right panel). (B) Expression of miR-153 was analyzed in two ovarian cancer cell lines (OVCAR3 and SKOV3) and normal ovarian cells (NOE095 and HOSEpiC). Data are presented as mean \pm SD. Expression of SET7 or ZEB2 was also measured in these cell lines. (C) Clinical data were plotted using Kaplan-Meier curves, and the 5-year survival rate was compared using the Cox log-rank test $\left({ }^{* *} \mathrm{P}<0.001\right)$. The $\mathrm{y}-\mathrm{axis}$ represents the survival probability, and the $\mathrm{x}$-axis represents the survival in months.

proved to play important roles in different cancers $(6,35,36)$. Accumulating evidence reveals the crucial role of miRNAs involved proliferation, invasion, prognosis and other biology processes (2). The concept of ZEB2 as powerful regulator of EMT has been widely accepted, and several miRNAs have also been described as crucial EMT regulators (19).

In the present study, the expression of miR-153 was lower in EOC tissues than that in the corresponding adjacent areas, and the upregulation of miR-153 inhibited the proliferation and invasion of OVCAR3 cell lines (Fig. 1C-E), while the effect of miR-153 overexpression was the reverse (Fig. 4B-D). Furthermore, we also discovered that the overexpression of miR-153 diminished but the knockdown of miR-153 increased SET7/ZEB2 expression level in OVCAR3 and SKOV3 cell lines. By directly binding the 3'-UTR of SET7/ZEB2, miR-153 promoted SET7/ZEB2 mRNA degradation. Ectopic miR-153 expression regulated the expression level of SET7 in the cell lines (OVCAR3 and SKOV3). SET7 and ZEB2 as potential targets of miR-153 in ovarian cancer was proved as miR-153 inhibited ovarian cancer proliferation and invasion via repression of SET7 and ZEB2 in vitro. Furthermore, we discovered that silencing of SET7 or ZEB2 partially abolished the enhancement of cell proliferation and invasion induced by downregulated miR-153, confirmed SET7 and ZEB2 as downstream target genes of miR-153. In the present study, we found that SET7 and ZEB2 were negatively associated with miR-153 in EOC tumor tissue and cell lines, higher expression of SET7 or CARM1 indicated a worse overall 5-year survival. On the contrary, the higher expression of miR-153 indicates a better overall 5-year survival. The above finding indicated that miR-153 contributes to regulating epithelial-to-mesenchymal transition (EMT). Since we are unsure whether miR-153 has other targets related to EOC proliferation and invasion, the changes in the development of EOC should not be attributed as the alteration of a small number of genes. Thus, further exploration is necessary of the potential role of miR-153 contribution to EOC. In future, miR-153-SET7 and miR-153-ZEB2 pathways may be exploited in a therapeutic approach for the treatment of ovarian cancer. 


\section{References}

1. Bartel DP: MicroRNAs: Target recognition and regulatory functions. Cell 136: 215-233, 2009.

2. Shukla GC, Singh J and Barik S: MicroRNAs: Processing, maturation, target recognition and regulatory functions. Mol Cell Pharmacol 3: 83-92, 2011.

3. Behm-Ansmant I, Rehwinkel J and Izaurralde E: MicroRNAs silence gene expression by repressing protein expression and/or by promoting mRNA decay. Cold Spring Harb Symp Quant Biol 71: 523-530, 2006.

4. Chen Y, Zhang L and Hao Q: Candidate microRNA biomarkers in human epithelial ovarian cancer: Systematic review profiling studies and experimental validation. Cancer Cell Int 13: 86, 2013.

5. Lu J, Getz G, Miska EA, Alvarez-Saavedra E, Lamb J, Peck D, Sweet-Cordero A, Ebert BL, Mak RH, Ferrando AA, et al: MicroRNA expression profiles classify human cancers. Nature 435: 834-838, 2005.

6. Wang JL, Hu Y, Kong X, Wang ZH, Chen HY, Xu J and Fang JY: Candidate microRNA biomarkers in human gastric cancer: A systematic review and validation study. PLoS One 8: e73683, 2013.

7. Gaur A, Jewell DA, Liang Y, Ridzon D, Moore JH, Chen C, Ambros VR and Israel MA: Characterization of microRNA expression levels and their biological correlates in human cancer cell lines. Cancer Res 67: 2456-2468, 2007.

8. Iorio MV and Croce CM: MicroRNA dysregulation in cancer: Diagnostics, monitoring and therapeutics. A comprehensive review. EMBO Mol Med 4: 143-159, 2012.

9. Esquela-Kerscher A and Slack FJ: Oncomirs-microRNAs with a role in cancer. Nat Rev Cancer 6: 259-269, 2006.

10. Reynolds EA and Moller KA: A review and an update on the screening of epithelial ovarian cancer. Curr Probl Cancer 30: 203-232, 2006

11. Ozols RF, Bookman MA, Connolly DC, Daly MB, Godwin AK, Schilder RJ, Xu X and Hamilton TC: Focus on epithelial ovarian cancer. Cancer Cell 5: 19-24, 2004.

12. Shan W and Liu J: Epithelial ovarian cancer: Focus on genetics and animal models. Cell Cycle 8: 731-735, 2009.

13. Farley J, Ozbun LL and Birrer MJ: Genomic analysis of epithelial ovarian cancer. Cell Res 18: 538-548, 2008.

14. Miyamoto M, Takano M, Goto T, Kato M, Sasaki N, Tsuda H and Furuya K: Clear cell histology as a poor prognostic factor for advanced epithelial ovarian cancer: A single institutional case series through central pathologic review. J Gynecol Oncol 24 37-43, 2013.

15. Shih IeM and Kurman RJ: Ovarian tumorigenesis: A proposed model based on morphological and molecular genetic analysis. Am J Pathol 164: 1511-1518, 2004.

16. Comijn J, Berx G, Vermassen P, Verschueren K, van Grunsven L, Bruyneel E, Mareel M, Huylebroeck D and van Roy F: The twohanded $\mathrm{E}$ box binding zinc finger protein SIP1 downregulates E-cadherin and induces invasion. Mol Cell 7: 1267-1278, 2001.

17. Vandewalle C, Comijn J, De Craene B, Vermassen P, Bruyneel E, Andersen H, Tulchinsky E, Van Roy F and Berx G: SIP1/ZEB2 induces EMT by repressing genes of different epithelial cell-cell junctions. Nucleic Acids Res 33: 6566-6578, 2005.

18. Ansieau S, Bastid J, Doreau A, Morel AP, Bouchet BP, Thomas C, Fauvet F, Puisieux I, Doglioni C, Piccinin S, et al: Induction of EMT by twist proteins as a collateral effect of tumor-promoting inactivation of premature senescence. Cancer Cell 14: 79-89, 2008
19. You J, Li Y, Fang N, Liu B, Zu L, Chang R, Li X and Zhou Q MiR-132 suppresses the migration and invasion of lung cancer cells via targeting the EMT regulator ZEB2. PLoS One 9: e91827, 2014

20. Peinado H, Olmeda D and Cano A: Snail, Zeb and bHLH factors in tumour progression: An alliance against the epithelial phenotype? Nat Rev Cancer 7: 415-428, 2007.

21. Gemmill RM, Roche J, Potiron VA, Nasarre P, Mitas M, Coldren CD, Helfrich BA, Garrett-Mayer E, Bunn PA and Drabkin HA: ZEB1-responsive genes in non-small cell lung cancer. Cancer Lett 300: 66-78, 2011.

22. Bindels S, Mestdagt M, Vandewalle C, Jacobs N, Volders L, Noël A, van Roy F, Berx G, Foidart JM and Gilles C: Regulation of vimentin by SIP1 in human epithelial breast tumor cells. Oncogene 25: 4975-4985, 2006.

23. Deering TG, Ogihara T, Trace AP, Maier B and Mirmira RG Methyltransferase Set7/9 maintains transcription and euchromatin structure at islet-enriched genes. Diabetes 58: 185-193, 2009.

24. Schneider R, Bannister AJ, Myers FA, Thorne AW, CraneRobinson C and Kouzarides T: Histone H3 lysine 4 methylation patterns in higher eukaryotic genes. Nat Cell Biol 6: 73-77, 2004.

25. Pradhan S, Chin HG, Estève PO and Jacobsen SE: SET7/9 mediated methylation of non-histone proteins in mammalian cells. Epigenetics 4: 383-387, 2009.

26. Tao Y, Neppl RL, Huang ZP, Chen J, Tang RH, Cao R, Zhang Y, Jin SW and Wang DZ: The histone methyltransferase Set7/9 promotes myoblast differentiation and myofibril assembly. J Cell Biol 194: 551-565, 2011.

27. Keating ST, Ziemann M, Okabe J, Khan AW, Balcerczyk A and El-Osta A: Deep sequencing reveals novel Set7 networks. Cell Mol Life Sci 71: 4471-4486, 2014.

28. Kouzarides T: Chromatin modifications and their function. Cell 128: 693-705, 2007.

29. Bracken CP, Li X, Wright JA, Lawrence DM, Pillman KA, Salmanidis M, Anderson MA, Dredge BK, Gregory PA, Tsykin A, et al: Genome-wide identification of miR-200 targets reveals a regulatory network controlling cell invasion. EMBO J 33: 2040-2056, 2014.

30. Pecot CV, Rupaimoole R, Yang D, Akbani R, Ivan C, Lu C, Wu S, Han HD, Shah MY, Rodriguez-Aguayo C, et al: Tumour angiogenesis regulation by the miR-200 family. Nat Commun 4: 2427, 2013.

31. Cheng W, Liu T, Wan X, Gao Y and Wang H: MicroRNA-199a targets $C D 44$ to suppress the tumorigenicity and multidrug resistance of ovarian cancer-initiating cells. FEBS J 279: 2047-2059, 2012.

32. Bartel DP: MicroRNAs: Genomics, biogenesis, mechanism, and function. Cell 116: 281-297, 2004.

33. Wu S, Huang S, Ding J, Zhao Y, Liang L, Liu T, Zhan R and He X: Multiple microRNAs modulate p21Cip1/Waf1 expression by directly targeting its 3 ' untranslated region. Oncogene 29 : 2302-2308, 2010.

34. Tili E, Michaille JJ and Croce CM: MicroRNAs play a central role in molecular dysfunctions linking inflammation with cancer. Immunol Rev 253: 167-184, 2013.

35. Yanokura M, Banno K, Kobayashi Y, Kisu I, Ueki A, Ono A, Masuda K, Nomura H, Hirasawa A, Susumu N, et al: MicroRNA and endometrial cancer: Roles of small RNAs in human tumors and clinical applications (Review). Oncol Lett 1: 935-940, 2010.

36. Zhao S, Deng Y, Liu Y, Chen X, Yang G, Mu Y, Zhang D, Kang J and $\mathrm{Wu} \mathrm{Z}$ : MicroRNA-153 is tumor suppressive in glioblastoma stem cells. Mol Biol Rep 40: 2789-2798, 2013. 